\title{
A score appraising Paleolithic diet and the risk of cardiovascular disease in a Mediterranean prospective cohort
}

\author{
Víctor de la $0^{1,4} \cdot$ Itziar Zazpe $^{1,2,3,4} \cdot$ Leticia Goni ${ }^{1,3,4} \cdot$ Susana Santiago ${ }^{2,4} \cdot$ Nerea Martín-Calvo $0^{1,3,4}$. \\ Maira Bes-Rastrollo ${ }^{1,3,4} \cdot$ J. Alfredo Martínez ${ }^{2,3,4,5} \cdot$ Miguel Á. Martínez-González ${ }^{1,3,4,6} \cdot$ Miguel Ruiz-Canela $^{1,3,4}(0)$
}

Received: 16 April 2021 / Accepted: 1 October 2021 / Published online: 21 October 2021

(c) The Author(s) 2021

\begin{abstract}
Purpose To assess the association between a score appraising adherence to the PaleoDiet and the risk of cardiovascular disease (CVD) in a Mediterranean cohort.

Methods We included 18,210 participants from the Seguimiento Universidad de Navarra (SUN) cohort study. The PaleoDiet score comprised six food groups promoted within this diet (fruit, nuts, vegetables, eggs, meat and fish) and five food groups whose consumption is discouraged (cereals and grains, dairy products, legumes, culinary ingredients, and processed/ultraprocessed foods). CVD was defined as acute myocardial infarction with or without ST elevation, non-fatal stroke and cardiovascular death. Cox proportional hazards models adjusted for potential confounders were fitted to assess the association between the PaleoDiet score and CVD risk, and the PaleoDiet and MedDiet indices to explore differences between both diets. Results During 12.2 years of follow-up, 165 incident CVD cases were confirmed. A significant inverse association was found between the PaleoDiet score and CVD (HR Q5 vs. Q1: 0.45, 95\% CI 0.27-0.76, P for trend =0.007). A weaker association that became non-significant was observed when the item for low consumption of ultra-processed foods was removed from the score. Joint analysis of PaleoDiet and MedDiet Trichopoulou scores suggested that the inverse association between PaleoDiet and CVD was mainly present when adherence to the MedDiet was also high (HR for high adherence vs low adherence to both diet scores: 0.22 , 95\% CI 0.08-0.64).
\end{abstract}

Conclusions Our findings suggest that the PaleoDiet may have cardiovascular benefits in participants from a Mediterranean country. Avoidance of ultra-processed foods seems to play a key role in this inverse association.

Keywords Paleolithic diet $\cdot$ Mediterranean diet $\cdot$ Cardiovascular risk $\cdot$ Dietary patterns $\cdot$ Cohort study

Miguel Ruiz-Canela

mcanela@unav.es

1 Department of Preventive Medicine and Public Health, School of Medicine, University of Navarra, Irunlarrea 1, 31008 Pamplona, Spain

2 Department of Nutrition, Food Sciences and Physiology, School of Pharmacy and Nutrition, University of Navarra, Pamplona, Spain

3 CIBER Fisiopatología de la Obesidad y Nutrición (CIBEROBN), Instituto de Salud Carlos III (ISCIII), Madrid, Spain

4 Navarra Institute for Health Research (IdiSNA), Pamplona, Spain

5 Precision Nutrition Program, IMDEA Food, CEI UAM + CSIC, Madrid, Spain

6 Department of Nutrition, Harvard TH Chan School of Public Health, Boston, USA

\begin{tabular}{ll}
\multicolumn{2}{l}{ Abbreviations } \\
BMI & Body mass index \\
CI & Confidence interval \\
CVD & Cardiovascular disease \\
FFQ & Food Frequency Questionnaire \\
HR & Hazard ratio \\
LDL & Low density lipoprotein \\
MDS & Mediterranean diet score \\
MEDAS & MEditerranean Diet Adherence Screener \\
METs & Metabolic equivalents of task \\
MedDiet & Mediterranean diet \\
MUFAs & Monounsaturated fatty acids \\
PaleoDiet & Paleolithic diet \\
PREDIMED & PREvención con DIeta MEDiterránea \\
P & Percentile \\
PUFAs & Polyunsaturated fatty acids \\
RR & Relative risk \\
SD & Standard deviation
\end{tabular}


SFAs

SUN

Saturated fatty acids

Seguimiento Universidad de Navarra

\section{Introduction}

Cardiovascular disease (CVD) remains the most common cause of death, accounting for 2.2 million deaths in females and 1.9 million deaths in males during 2019 in Europe, and $31 \%$ of all deaths globally $[1,2]$. Moreover, CVD incidence is globally increasing due to population growth, rural-tourban migration and the aging of the world's population [3]. CVD-related morbidity is associated with a gradual decrease in the quality of life and higher economic cost [4, 5], and it accounts for $24 \%$ of non-communicable diseases related disability-adjusted life years globally [5]. The promotion of healthy lifestyles to prevent CVD is an urgent need to reduce the current burden of this public health concern [6].

The promotion of healthy diets is probably one of the most cost-effective strategies to prevent CVD. Several dietary patterns have been proposed based on the cardioprotective effect of their components [7]. The Mediterranean Diet (MedDiet), the Alternative Healthy Eating Index or the Dietary Approaches to Stop Hypertension are well-known dietary patterns. They represent a priori defined high-quality diet scores inversely associated with the risk of CVD [8]. A common characteristic of these dietary patterns is a high consumption of fruits and vegetables, whole grains, nuts, legumes, vegetable oils, fish, and seafood; a moderate consumption of low-fat dairy products; and a low consumption of processed meat, sugar-sweetened beverages, and sodium [9].

Currently, there is an emergence of additional alternative dietary patterns with attributed health benefits although they usually rely only on limited scientific evidence. The Paleolithic diet (PaleoDiet) or "Diet Hunter-Gatherer" promotes the consumption of wild animal and plant food according to the supposed lifestyles of humankind during the Paleolithic age [10]. The PaleoDiet has increased its popularity during the last years [11], especially among young adults and athlete population, but also in patients with chronic diseases such as inflammatory bowel disease and celiac disease [12-14].

Anthropological studies have hypothesized that huntergatherers had a slim build, and they were fit and free of chronic diseases such as CVD due to their diet $[15,16]$. From a public health perspective, the challenge is to know the potential health benefits of a Paleo-style dietary pattern adapted to current lifestyles and food availability. Currently, the PaleoDiet is characterized by a high consumption of fruits, vegetables, tree nuts, eggs, fish, and unprocessed meats, and a low consumption of dairy products, cereals and grains, legumes, processed foods, and culinary ingredients (added salt, sugar and refined fats) [17]. However, there is a diverse interpretation about the PaleoDiet at the popular levels and these Paleo-style diets may have no clear definitions and there is scarce scientific evidence to promote them [17]. Some recent reviews have suggested an inverse association between the PaleoDiet and cardiovascular risk factors $[17,18]$. However, there is scarce evidence on the long-term beneficial effect of the PaleoDiet on hard end-points of the most prevalent chronic diseases such as CVD. To the best of our knowledge, only two studies with large sample size have suggested potential benefits of the PaleoDiet on CVD mortality $[19,20]$.

In this study we aimed to assess the association between a score appraising a PaleoDiet pattern and the risk of CVD in a well-known Spanish cohort. Knowing the association between this dietary pattern and CVD risk in a country with a relatively high adherence to the MedDiet is of interest. For this reason, we also explored the relationship between the MedDiet and PaleoDiet. While the MedDiet emphasizes a high consumption of all plant-based foods, the PaleoDiet limits the intake of legumes, cereals and grains, and recommends higher intake of unprocessed meats. Our hypothesis was that the PaleoDiet could reduce CVD risk due to the high consumption of fruits, vegetables, tree nuts, eggs, fish, unprocessed meats, and the exclusion of ultra-processed foods. In addition, we hypothesized that the recommendation to reduce the consumption of whole grains and legumes within the PaleoDiet score might mitigate the inverse association between this diet and CVD risk.

\section{Subjects and methods}

\section{Study design and population}

The Seguimiento Universidad de Navarra (SUN) study is a dynamic multipurpose prospective cohort of Spanish university graduates. A detailed description of the design and methodology is available elsewhere [21]. Briefly, selfreported questionnaires at baseline allowed to collect information about sociodemographic characteristics, lifestyle and eating habits, and health conditions of the participants. Information about some lifestyle factors and health outcomes has been updated biennially.

The SUN cohort started in 1999 and since then, the total number of recruited participants until December 2019 was 22,894 . From them, 22,553 participants were eligible for analyses of incident CVD to ensure that they could complete at least the first follow-up questionnaire. Additionally, we excluded 350 participants with prevalent CVD (myocardial infarction and revascularization), 2114 participants with total energy intake outside predefined limits $(<500$ or $>3500 \mathrm{kcal} /$ day for females, or $<800$ or $>4000 \mathrm{kcal} /$ day 
for males) [22], 1671 without follow-up information, and 208 with more than $50 \%$ of missing items in the semi-quantitative Food Frequency Questionnaire (FFQ). Therefore, 18,210 participants were included in our analyses (Fig. 1).

\section{Ethics}

Participants received written information about the specific data required in the questionnaires, the protection to safeguard their privacy, and the future feedback from the research team. We also informed the potential candidates of their right to refuse to participate in the SUN study or to withdraw their consent to participate at any time without reprisal, according to the principles of the Declaration of Helsinki. The voluntary completion of the baseline questionnaire was considered to imply informed consent. The Research Ethics Committee of the University of Navarra approved this method to request the informed consent of participants. This cohort is registered at clinicaltrials.gov as NCT02669602.

\section{Dietary assessment}

A validated 136-item semi-quantitative FFQ was used at baseline to collect information about diet [21, 23, 24]. Consumption frequencies were grouped into nine categories ranging from never/almost never to $6+$ times per day. Total energy and nutrient intake were calculated using Spanish food composition tables $[25,26]$. We multiplied typical portion sizes by consumption frequency for each food item to calculate the daily food consumption.

Ultra-processed foods were defined according to the classification system based on the extent and purpose of industrial food processing (NOVA food groups) [27]. Ultraprocessed foods are defined as foods or drink products with low nutrient density and/or high energy density. The fourth
Fig. 1 Flow-chart of participants recruited in the SUN Project, 1999-2019

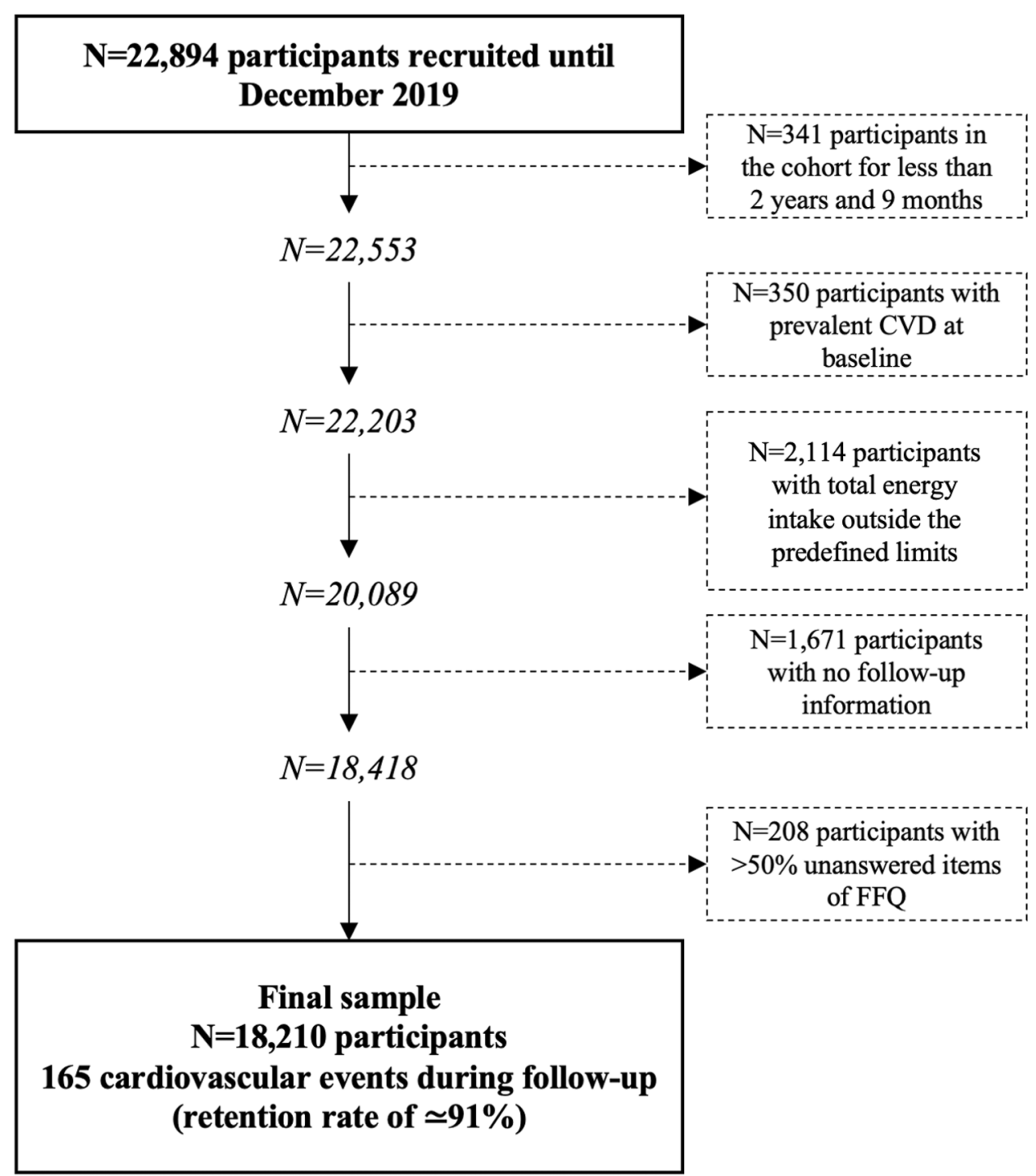


NOVA category includes ultra-processed food and beverage that are typically created by series of industrial techniques and processes. Sugar, oils and fats, salt and additives that prolong product duration used to make processed foods, are often ingredients of ultra-processed foods, commonly in combination [27]. These products are usually ready for consumption such as carbonated drinks, sausages, biscuits and cookies, candies (confectionery), instant packaged soups and noodles, sweet or savory packaged snacks, and sugared milk and fruit drinks [27, 28]. To estimate the frequency of consumption of ultra-processed food we summed the amount consumed (servings per day) of each food item classified in the fourth category of the NOVA system (Supplemental Table 1). More detailed methodology has been described in previous studies conducted in the SUN cohort [28-30].

\section{The PaleoDiet score}

The adherence to the PaleoDiet was assessed according to a published score that followed the theoretical definition of that dietary pattern according to previous definitions [17]. This index included six food groups promoted within the PaleoDiet (fruits, nuts, vegetables, eggs, meat and fish) and five food groups whose consumption is discouraged in this dietary model (cereals and grains, dairy products, legumes, culinary ingredients (added sugars, salt and refined fats) and processed/ultra-processed foods). We gave the same weight to all items and accordingly each one accounted for a maximum of five points in our score. All foods that comprised each group are shown in Supplemental Table 1. For each food group, participants were categorized into quintiles. Each quintile was assigned a numeric value ranging from 1 to 5 (an inverse punctuation was used for non-characteristic components of the PaleoDiet). The sum of all the components resulted in the PaleoDiet score with a theoretically minimum value of 11 and a maximum of 55 .

\section{Assessment of Mediterranean Diet}

We used two a priori defined MedDiet indices to assess the level of adherence to the traditional Mediterranean dietary pattern, which is characterized by a high intake of olive oil, vegetables, legumes, fruits, nuts, whole cereals, and fish; moderate consumption of red wine; low consumption of lean meat and dairy products, and very low or null consumption of red and processed meat. First, we used the Mediterranean Diet Score (MDS) developed by Trichopoulou and cols. (a widely used 9-unit scale based on nine dietary components that captures the essence of the traditional MedDiet) [31]. Second, we applied the 14-point Mediterranean Diet adherence Screener (MEDAS) used in the Prevención con Dieta Mediterránea (PREDIMED) trial, which includes five additional items that are also critical to an accurate assessment of the adherence to the traditional MedDiet, and is validated with objective biomarkers [32, 33]. Differences of these two MedDiet scores are briefly described in Supplemental Table 2.

\section{Outcome assessment}

The endpoint was a composite of acute myocardial infarction with or without ST elevation, non-fatal stroke (both confirmed by a review of medical records with prior permission of relatives) and cardiovascular death. When a CVD was self-reported in a follow-up questionnaire, complete medical information was requested to the participant to confirm the CVD diagnosis by a cardiologist who was blind to diet and lifestyle exposure. In addition, we consulted the National Death Index annually to identify the deceased participants and to obtain their cause of death. In some cases, information about participants' death was obtained from participants' relatives, postal authorities and work associates.

\section{Statistical analysis}

Baseline characteristics are described as mean \pm standard deviation (SD) for quantitative variables and percentages for categorical variables. All variables shown in Tables 1 and 2 were age- and sex-adjusted using the Inverse Probability Weighting method [34]. Standard tests were applied to assess differences between the means and proportions of cohort characteristics according to PaleoDiet score quintiles.

We first analyzed the PaleoDiet score as a continuous variable and then categorized it into quintiles to define low $(\mathrm{Q} 1)$, low-moderate $(\mathrm{Q} 2)$, moderate $(\mathrm{Q} 3)$, high-moderate (Q4) and high (Q5) adherence to the PaleoDiet. Cox proportional hazard regression models were used to estimate Hazard Ratios (HR) for CVD and their 95\% confidence intervals (95\% CI). We used the lowest quintile as the reference value and we also calculated the HRs for 5-unit increase in the PaleoDiet score.

In addition, we estimated the relative importance of each of the components of the PaleoDiet score by subtracting alternately one component from the original score, and afterwards estimating the HRs per 5-unit increment in the score. The comparability between the scores was preserved by multiplying the logarithm of the estimated HRs by $50 / 55$ before exponentiating them (estimated HR for a 5-unit increment in PaleoDiet score $=e(5 \times[\beta$ coefficient of the PaleoDiet score without "item"] $\times(50 / 55))$ [35].

A crude model and three multivariable-adjusted models were fitted. Age was used as the underlying timevariable in all Cox models. In model 1 we adjusted for sex, and we stratified for age (deciles) and year entering the cohort (1999-2001, 2002-2004, 2005-2007, 2008-2010, 2011-2014, 2015-2017) using the option 
Table 1 Age and sex-adjusted baseline characteristics of the participants of the SUN cohort according to quintiles of the PaleoDiet score (19992017)

\begin{tabular}{|c|c|c|c|c|c|}
\hline & \multicolumn{5}{|c|}{ Quintiles of the Paleolithic diet score (min-max) } \\
\hline & Q1 & Q2 & Q3 & Q4 & Q5 \\
\hline Score, $\min -\max$ & $(16-28)$ & $(29-30)$ & $(31-33)$ & $(34-36)$ & $(37-50)$ \\
\hline$N$ & 4606 & 2699 & 4269 & 3515 & 3121 \\
\hline BMI, $\mathrm{kg} / \mathrm{m}^{2}$ & $23.4 \pm 3.6$ & $23.5 \pm 3.5$ & $23.4 \pm 3.4$ & $23.7 \pm 3.6$ & $23.7 \pm 3.6$ \\
\hline Education level, years & $5.1 \pm 1.5$ & $5.1 \pm 1.5$ & $5.1 \pm 1.5$ & $5.1 \pm 1.5$ & $5 \pm 1.5$ \\
\hline \multicolumn{6}{|l|}{ Smoking, $\%$} \\
\hline Non-smokers & 47.5 & 48 & 49.3 & 48.8 & 49.7 \\
\hline Former smokers & 27.1 & 27.3 & 28.8 & 30.4 & 30.5 \\
\hline Current smokers & 24.6 & 24.1 & 21.2 & 20.1 & 18.9 \\
\hline \multicolumn{6}{|l|}{ Prevalent disease, $\%$} \\
\hline Hypertension & 5.9 & 7.3 & 6.3 & 7.7 & 7.6 \\
\hline Hypertriglyceridemia & 6.0 & 6.4 & 6.6 & 7.4 & 7.1 \\
\hline Hypercholesterolemia & 16.8 & 17.2 & 16.9 & 16.9 & 17.0 \\
\hline Type 2 diabetes & 0.8 & 1.3 & 1.7 & 1.9 & 2.9 \\
\hline Cancer & 2.4 & 2.7 & 2.4 & 2.3 & 2.6 \\
\hline Depression & 11.7 & 12.4 & 11.1 & 11.6 & 11.2 \\
\hline Napping, $\%$ & 55.2 & 55.2 & 53.1 & 54.7 & 56.1 \\
\hline Snacking between meals, $\%$ & 35.7 & 33.6 & 33.5 & 31.7 & 31.1 \\
\hline Special diets, $\%$ & 4.8 & 6.0 & 7.2 & 10.1 & 13.9 \\
\hline Watching television, $\mathrm{h} /$ day & $1.7 \pm 1.2$ & $1.6 \pm 1.2$ & $1.6 \pm 1.2$ & $1.6 \pm 1.2$ & $1.5 \pm 1.1$ \\
\hline Siting, h/day & $5.3 \pm 2.1$ & $5.3 \pm 2.0$ & $5.3 \pm 2.1$ & $5.2 \pm 2.0$ & $5.2 \pm 2.1$ \\
\hline Physical activity, METs-h/week & $18.7 \pm 20.4$ & $19.8 \pm 21.8$ & $22.1 \pm 23.2$ & $23.6 \pm 23.3$ & $26.6 \pm 27.1$ \\
\hline Mediterranean diet score (MDS) [31] & $3.7 \pm 1.6$ & $4.0 \pm 1.8$ & $4.3 \pm 1.8$ & $4.7 \pm 1.7$ & $5.1 \pm 1.6$ \\
\hline $\begin{array}{l}\text { Mediterranean Diet Adherence Screener } \\
\text { (MEDAS) [32] }\end{array}$ & $5.0 \pm 1.6$ & $5.6 \pm 1.6$ & $6.1 \pm 1.7$ & $6.6 \pm 1.7$ & $7.3 \pm 1.7$ \\
\hline Fruits, g/day & $234.3 \pm 215.6$ & $297.4 \pm 258.3$ & $343.3 \pm 282.3$ & $408.5 \pm 306.2$ & $490.2 \pm 337.4$ \\
\hline Nuts, g/day & $3.9 \pm 6.7$ & $5 \pm 7.7$ & $6.9 \pm 10.8$ & $9.3 \pm 13.6$ & $13 \pm 16.3$ \\
\hline Fish, g/day & $70.2 \pm 43.9$ & $84.6 \pm 48.6$ & $97.7 \pm 58.8$ & $113.2 \pm 62.6$ & $135.1 \pm 62.6$ \\
\hline Eggs, g/day & $21.1 \pm 15.8$ & $22.1 \pm 14$ & $23 \pm 14$ & $24.6 \pm 16.7$ & $26.5 \pm 19.6$ \\
\hline Vegetables, g/day & $364.4 \pm 230.1$ & $463.2 \pm 289.9$ & $528.4 \pm 319.6$ & $623 \pm 360.4$ & $732.2 \pm 371.6$ \\
\hline Olive oil, g/day & $17.5 \pm 15.2$ & $18.4 \pm 14.6$ & $18.6 \pm 14.9$ & $19.3 \pm 14.8$ & $19.6 \pm 14.8$ \\
\hline \multicolumn{6}{|l|}{ Unprocessed meats, g/day } \\
\hline Lean meat & $37.3 \pm 31.4$ & $41.3 \pm 29.1$ & $46.2 \pm 33.1$ & $51.3 \pm 35.9$ & $60.7 \pm 42.5$ \\
\hline Red meat & $80.5 \pm 44.6$ & $89.2 \pm 47.9$ & $94.1 \pm 50.6$ & $98.1 \pm 51.7$ & $104.1 \pm 56.7$ \\
\hline Cereals and grains, g/day & $119.2 \pm 79.4$ & $102 \pm 70.8$ & $94.3 \pm 67.6$ & $85.3 \pm 66$ & $70.3 \pm 56.7$ \\
\hline Dairy products, g/day & $469.2 \pm 262.7$ & $423.3 \pm 251.9$ & $390.1 \pm 247.2$ & $368.2 \pm 250.3$ & $318 \pm 244.3$ \\
\hline Legumes, g/day & $25 \pm 18.8$ & $22.7 \pm 17.6$ & $22.9 \pm 17.5$ & $22.3 \pm 18.2$ & $20.7 \pm 17.2$ \\
\hline Ultra-processed food, g/day & $345.8 \pm 204.8$ & $314.5 \pm 196.5$ & $295.7 \pm 190.6$ & $280.8 \pm 185.4$ & $240.6 \pm 159.3$ \\
\hline \multicolumn{6}{|l|}{ Culinary ingredients, g/day } \\
\hline Added salt & $1.6 \pm 1.4$ & $1.6 \pm 1.5$ & $1.5 \pm 1.4$ & $1.5 \pm 1.4$ & $1.3 \pm 1.4$ \\
\hline Added sugar & $19 \pm 17.3$ & $14.3 \pm 15$ & $11.2 \pm 13.5$ & $8.4 \pm 11.8$ & $5.6 \pm 9.9$ \\
\hline Refined fats & $4.1 \pm 7.9$ & $3.1 \pm 6.1$ & $2.9 \pm 6.6$ & $2.4 \pm 5.9$ & $1.7 \pm 4.9$ \\
\hline Sugar-sweetened beverages, $\mathrm{ml} /$ day & $56.4 \pm 106.5$ & $43.8 \pm 78.9$ & $38.1 \pm 71.3$ & $32.8 \pm 73.3$ & $20.8 \pm 44$ \\
\hline
\end{tabular}

Means \pm SD are shown unless otherwise stated. Table adjusted for age and sex by the Inverse Probability Weighting method

$B M I$ body mass index, $M E T$ metabolic equivalent of task, $Q$ quintile 
Table 2 Age and sex-adjusted baseline nutritional values according to quintiles of the PaleoDiet score in participants of the SUN cohort (19992017)

\begin{tabular}{|c|c|c|c|c|c|}
\hline & \multicolumn{5}{|c|}{ Quintiles of the Paleolithic diet score (min-max) } \\
\hline & Q1 & Q2 & Q3 & Q4 & Q5 \\
\hline PaleoDiet Score, min-max & $(16-28)$ & $(29-30)$ & $(31-33)$ & $(34-36)$ & $(37-50)$ \\
\hline$N$ & 4606 & 2699 & 4269 & 3515 & 3121 \\
\hline Total energy, kcal/day & $2421 \pm 599$ & $2349 \pm 623$ & $2331 \pm 620$ & $2343 \pm 626$ & $2311 \pm 590$ \\
\hline \multicolumn{6}{|l|}{ Macronutrients } \\
\hline Carbohydrate intake, $\%$ of TEI & $46 \pm 70$ & $44.1 \pm 6.8$ & $43.1 \pm 7.1$ & $42.1 \pm 7.4$ & $40.6 \pm 7.6$ \\
\hline Protein intake, $\%$ of TEI & $16.6 \pm 2.7$ & $17.6 \pm 2.7$ & $18.3 \pm 2.9$ & $19 \pm 3.2$ & $20 \pm 3.4$ \\
\hline Animal protein & $11.5 \pm 3.0$ & $12.4 \pm 3.0$ & $12.9 \pm 3.2$ & $13.5 \pm 3.5$ & $14.3 \pm 3.7$ \\
\hline Vegetal protein & $5.2 \pm 1.2$ & $5.2 \pm 1.2$ & $5.4 \pm 1.3$ & $5.5 \pm 1.3$ & $5.7 \pm 1.4$ \\
\hline Fat intake, $\%$ of TEI & $35.5 \pm 6.5$ & $36.2 \pm 6.4$ & $36.6 \pm 6.5$ & $36.9 \pm 6.5$ & $37.4 \pm 6.6$ \\
\hline SFA intake, $\%$ of TEI & $12.6 \pm 3.1$ & $12.6 \pm 3.1$ & $12.5 \pm 3.1$ & $12.2 \pm 3.2$ & $12.0 \pm 3.2$ \\
\hline MUFA intake, $\%$ of TEI & $14.9 \pm 3.5$ & $15.5 \pm 3.5$ & $15.8 \pm 3.7$ & $16 \pm 3.7$ & $16.4 \pm 3.8$ \\
\hline PUFA intake, $\%$ of TEI & $5.1 \pm 1.7$ & $5.1 \pm 1.5$ & $5.1 \pm 1.5$ & $5.2 \pm 1.4$ & $5.3 \pm 1.4$ \\
\hline n-3 fatty acids, g/day & $2.3 \pm 1.3$ & $2.4 \pm 1.1$ & $2.6 \pm 1.2$ & $2.8 \pm 1.2$ & $3.1 \pm 1.2$ \\
\hline n-6 fatty acids, g/day & $19.8 \pm 13.8$ & $18.3 \pm 12.2$ & $17.3 \pm 11.1$ & $16.9 \pm 10.6$ & $15.8 \pm 9.4$ \\
\hline Fiber intake, g/day & $23.5 \pm 9.6$ & $25.7 \pm 11.1$ & $27.8 \pm 11.7$ & $30.6 \pm 12.7$ & $34.2 \pm 13.2$ \\
\hline Alcohol intake, g/day & $6.2 \pm 9.4$ & $6.7 \pm 10.5$ & $6.8 \pm 10.8$ & $6.6 \pm 9.5$ & $6.7 \pm 10.2$ \\
\hline \multicolumn{6}{|l|}{ Micronutrients } \\
\hline Fe, mg/day & $15.4 \pm 4.3$ & $16.2 \pm 4.8$ & $17 \pm 5$ & $18.1 \pm 5.2$ & $19.3 \pm 5.3$ \\
\hline $\mathrm{Cr}, \mu \mathrm{g} /$ day & $93.5 \pm 41.7$ & $87.7 \pm 36.2$ & $87 \pm 36.3$ & $86.1 \pm 36.4$ & $83.6 \pm 32.3$ \\
\hline $\mathrm{I}, \mu \mathrm{g} / \mathrm{day}$ & $377.5 \pm 219.5$ & $346.8 \pm 212.4$ & $322.5 \pm 192.6$ & $313.9 \pm 199.2$ & $286 \pm 183.7$ \\
\hline $\mathrm{Mg}, \mathrm{mg} /$ day & $382.9 \pm 105.4$ & $396.2 \pm 116.5$ & $413.8 \pm 122.2$ & $438 \pm 128$ & $466.6 \pm 129.4$ \\
\hline $\mathrm{Ca}, \mathrm{mg} /$ day & $1243 \pm 460.8$ & $1222 \pm 478.3$ & $1219 \pm 478.2$ & $1234 \pm 472.8$ & $1221 \pm 472.6$ \\
\hline $\mathrm{P}, \mathrm{mg} / \mathrm{day}$ & $1862 \pm 501.9$ & $1883 \pm 521.9$ & $1922 \pm 545$ & $1983 \pm 550.9$ & $2034 \pm 551.4$ \\
\hline $\mathrm{K}, \mathrm{mg} /$ day & $4148 \pm 1242$ & $4480 \pm 1443$ & $4755 \pm 1525$ & $5167 \pm 1622$ & $5623 \pm 1659$ \\
\hline $\mathrm{Na}, \mathrm{mg} /$ day & $3659 \pm 2347$ & $3402 \pm 2179$ & $3296 \pm 2552$ & $3198 \pm 1970$ & $2950 \pm 1680$ \\
\hline $\mathrm{Na} / \mathrm{K}$ ratio & $0.92 \pm 0.61$ & $0.79 \pm 0.53$ & $0.72 \pm 0.53$ & $0.64 \pm 0.40$ & $0.54 \pm 0.32$ \\
\hline $\mathrm{Se}, \mu \mathrm{g} /$ day & $89.9 \pm 33.9$ & $91.6 \pm 32.6$ & $95 \pm 33.6$ & $99.4 \pm 35.3$ & $105.2 \pm 35.7$ \\
\hline $\mathrm{Zn}, \mathrm{mg} / \mathrm{day}$ & $16.4 \pm 9.9$ & $17.2 \pm 10.2$ & $17.9 \pm 11.3$ & $19.1 \pm 11.2$ & $19.7 \pm 12.4$ \\
\hline Vitamin A, $\mu \mathrm{g} /$ day & $1405 \pm 996$ & $1747 \pm 1357$ & $1964 \pm 1321$ & $2294 \pm 1593$ & $2690 \pm 1645$ \\
\hline Vitamin B1, mg/day & $1.7 \pm 0.5$ & $1.8 \pm 0.5$ & $1.8 \pm 0.6$ & $1.9 \pm 0.6$ & $20 \pm 0.6$ \\
\hline Vitamin B2, mg/day & $2.2 \pm 0.7$ & $2.2 \pm 0.7$ & $2.2 \pm 0.7$ & $2.3 \pm 0.7$ & $2.3 \pm 0.7$ \\
\hline Vitamin B3, mg/day & $37.6 \pm 10.1$ & $39.9 \pm 10.7$ & $42.2 \pm 11.5$ & $44.9 \pm 11.9$ & $48.6 \pm 12.2$ \\
\hline Vitamin B6, mg/day & $2.3 \pm 0.7$ & $2.5 \pm 0.8$ & $2.8 \pm 0.9$ & $3.0 \pm 0.9$ & $3.4 \pm 1.0$ \\
\hline Vitamin B12, $\mu \mathrm{g} /$ day & $7.9 \pm 4.0$ & $8.8 \pm 4.3$ & $9.5 \pm 5.0$ & $10.3 \pm 4.9$ & $11.7 \pm 5.9$ \\
\hline Vitamin C, mg/day & $212.6 \pm 110.6$ & $253.9 \pm 134.3$ & $282 \pm 146.7$ & $318.9 \pm 159.8$ & $366.6 \pm 174.3$ \\
\hline Vitamin E, mg/day & $6.5 \pm 3.7$ & $6.6 \pm 3.3$ & $6.8 \pm 3.4$ & $7.2 \pm 3.5$ & $7.8 \pm 3.7$ \\
\hline Folic acid, $\mu \mathrm{g} / \mathrm{day}$ & $331.2 \pm 133.9$ & $375.6 \pm 156.9$ & $410.9 \pm 168.8$ & $454.1 \pm 176.8$ & $512.3 \pm 186.2$ \\
\hline
\end{tabular}

Means \pm SD are shown unless otherwise stated. Table adjusted for age and sex by the Inverse Probability Weighting method

MUFA monounsaturated fatty acids, PUFA polyunsaturated fatty acids, $Q$ quintile, TEI total energy intake, SFAs saturated fatty acids

"strata" in STATA. In model 2 we additionally adjusted for total energy intake (continuous), alcohol intake (abstainer, $>0$ to $\leq 5 \mathrm{~g} /$ day, $>5$ to $\leq 25 \mathrm{~g} / \mathrm{day}$ and $>25 \mathrm{~g} /$ day among females; abstainer, $>0$ to $\leq 10 \mathrm{~g} /$ day, $>10$ to $\leq 50 \mathrm{~g} /$ day and $>50 \mathrm{~g} /$ day among males), smoking status (non-smoker, former smoker, current smoker), packyears of cigarette smoking (continuous), body mass index
(BMI) (continuous), physical activity (METs-h/week as continuous), years of university education (continuous), family history of CVD (yes/no) and self-reported prevalent diseases at baseline, including hypertension, hypertriglyceridemia, hypercholesterolemia, diabetes, cancer, and depression. Finally, in model 3 we additionally adjusted for squared BMI, mid-day napping (yes/ 
no), watching television (h/day), sitting time (h/week), between-meal snacking (yes/no) and following special diets (yes/no). To investigate linear trends across the quintiles of PaleoDiet scores we assigned the median value to each quintile and considered the variable as continuous.

We calculated Pearson correlation coefficients $(r)$ to assess the strength and direction of the association between the PaleoDiet and the two MedDiet indices. To assess the potential effect of PaleoDiet in participants within a Mediterranean country, we conducted a joint analysis for the combination of MedDiet (using the MDS and MEDAS scores) and PaleoDiet scores. Both the MDS and PaleoDiet scores were divided into three categories $(\mathrm{Q} 1$, merged Q2-Q3-Q4, and Q5). Therefore, a joint variable with nine categories was created. The joint analysis for PaleoDiet and MEDAS were divided into three categories (Q1, merged Q2-Q3-Q4, and Q5) for PaleoDiet and into two categories according to the median for the MEDAS. Therefore, a joint variable with six categories was created. Radar plot is a useful technique for the graphic presentation of multivariate data [36]. We applied radar plots according to the joint analysis categories of PaleoDiet and MDS score to observe the standardized mean intake of the food groups used to define the PaleoDiet. The vertexes of the radar plot show the characteristic (dark light line) and non-characteristic (thick longitudinal line) foods of the PaleoDiet score.

Cox proportional hazard regression models were used to estimate the association between the joint categories of the PaleoDiet and MedDiet scores and CVD incidence, using the category with the lowest quintile for both MedDiet and PaleoDiet as the reference category.

We additionally conducted stratified analyses by sex, BMI ( $<25 \mathrm{~kg} / \mathrm{m}^{2}$ vs. $\left.\geq 25 \mathrm{~kg} / \mathrm{m}^{2}\right)$, and leisure-time physical activity ( $\leq 20$ METs-h/week vs. $>20$ METs-h/week). Likelihood-ratio tests were applied to assess potential effect modification by these variables. We also conducted joint analysis for BMI $\left(<25\right.$ and $\left.\geq 25 \mathrm{~kg} / \mathrm{m}^{2}\right)$ and physical activity $(\leq 20$ and $>20$ METs-h per week) with adherence to the PaleoDiet score, again recategorized into 3 categories, Q1 (low adherence, as reference), merged Q2-Q3-Q4 (intermediate adherence), and Q5 (high adherence).

Finally, to assess the robustness of our findings we conducted the following sensitivity analyses: including self-reported but non-confirmed CVD events as incident cases; modifying limits of total energy < percentile 1 (P1) and $>$ percentile 99 (P99) (i.e., excluding participants with total energy intake $<1073$ or $>3777 \mathrm{kcal} /$ day); excluding participants with a special diet at baseline; excluding participants $<40$ years; excluding participants with chronic aspirin intake; excluding participants with hypertension at baseline; and additionally excluding participants with other causes of CVD different from the outcome prevalent at baseline (aneurysm of the aorta, heart failure, atrial fibrillation, pulmonary embolism, peripheral venous thrombosis and intermittent claudication).

We used STATA software to conduct all the analyses (STATA version 14.0, StataCorp, College Station, TX, USA). All $P$ values presented are two-tailed. Statistical significance was considered at the conventional 0.05 level.

\section{Results}

Among 18,210 participants (60.6\% females), mean age at baseline was $38 \pm 12$ years old. After a median follow-up of 12.2 years (209,867 person-years), we ascertained 165 incident cases of CVD (80 non-fatal myocardial infarctions, 60 non-fatal strokes, and 25 cardiovascular deaths).

Age- and sex-adjusted baseline characteristics of participants according to quintiles of the PaleoDiet score are summarized in Table 1. Differences in baseline characteristics according to quintiles of the PaleoDiet score were statistically significant $(P<0.001)$ except for education level $(P=0.763)$ and calcium intake $(P=0.106)$. Higher prevalent type 2 diabetes, and lower percentage of current smokers were observed across quintiles of the PaleoDiet score. Participants in the highest quintile practiced more physical activity, reported higher adherence to the MedDiet, and were more likely to follow special diets. Whereas, they were less likely to consume snacks between meals and watching television. As expected, participants across successive quintiles of the PaleoDiet score consumed more fruits, nuts, fish, eggs, vegetables, olive oil and unprocessed meats, and fewer cereals and grains, dairy products, legumes, ultra-processed foods, culinary ingredients and sugar-sweetened beverages.

Baseline age and sex-adjusted energy and nutrient intakes according to adherence to the PaleoDiet score are displayed at Table 2. Participants in the highest quintile referred the lowest total daily energy and carbohydrate intakes, but highest total protein (animal and plant source) and fat intake (monounsaturated (MUFA), polyunsaturated (PUFA) and n-3 fatty acids; and a lower intake of saturated (SFA) and n-6 fatty acids). As expected, participants with higher adherence to PaleoDiet consumed more fiber, $\mathrm{Fe}, \mathrm{K}, \mathrm{Mg}, \mathrm{P}, \mathrm{Se}$, $\mathrm{Zn}$, vitamin $\mathrm{A}$, all vitamins from group $\mathrm{B}$, vitamin $\mathrm{C}$ and vitamin $\mathrm{E}$ than those with lower adherence. In addition, a lower $\mathrm{Na} / \mathrm{K}$ ratio was observed in the higher quintiles of the PaleoDiet score.

Table 3 shows a significant inverse association between the highest quintile of the PaleoDiet score and CVD risk compared to the lowest quintile in all regression models. A significant inverse association was also found for Q3 but not for Q4, although the $P$ for linear trend was statistically significant in all models. A 19\% (95\% CI 32-4\%) reduced 
Table 3 Relative risk of cardiovascular disease (hazard ratios) according to quintiles of the PaleoDiet score among participants of the SUN cohort (1999-2017)

\begin{tabular}{|c|c|c|c|c|c|c|c|}
\hline & \multicolumn{6}{|c|}{ Quintiles of Paleolithic diet score (min-max) } & \multirow[t]{2}{*}{$\mathrm{HR}(95 \% \mathrm{CI})$} \\
\hline & (Q1) & $(\mathrm{Q} 2)$ & (Q3) & (Q4) & (Q5) & $P$ trend $^{\mathrm{a}}$ & \\
\hline Limits & $16-28$ & $29-30$ & $31-33$ & $34-36$ & $37-50$ & & Per 5-unit increment \\
\hline Cases/person-y & $42 / 55,306$ & $22 / 31,460$ & $31 / 49,231$ & $42 / 39,941$ & $28 / 33,985$ & & \\
\hline Crude & 1 (Ref.) & $0.69(0.41,1.16)$ & $0.50(0.31,0.80)$ & $0.65(0.42,1.01)$ & $0.42(0.26,0.68)$ & 0.001 & $0.78(0.66-0.93)$ \\
\hline Model 1 & 1 (Ref.) & $0.72(0.43,1.23)$ & $0.50(0.31,0.81)$ & $0.72(0.46,1.13)$ & $0.47(0.28,0.78)$ & 0.008 & $0.81(0.68-0.96)$ \\
\hline Model 2 & 1 (Ref.) & $0.69(0.40,1.19)$ & $0.50(0.30,0.82)$ & $0.70(0.44,1.12)$ & $0.44(0.26,0.73)$ & 0.005 & $0.80(0.67-0.95)$ \\
\hline Model 3 & 1 (Ref.) & $0.69(0.40,1.19)$ & $0.51(0.31,0.83)$ & $0.72(0.45,1.15)$ & $0.45(0.27,0.76)$ & 0.007 & $0.81(0.68-0.96)$ \\
\hline
\end{tabular}

Crude model adjusted for age (10 groups) as the underlying variable of time. Model 1 adjusted for sex, stratified for age deciles and year entering the cohort (1999-2001, 2002-2004, 2005-2007, 2008-2010, 2011-2014, 2015-2017). Model 2 additionally adjusted for total energy intake (continuous), alcohol intake (teetotaler, $>0-5 \mathrm{~g} /$ day in females and $>0-10 \mathrm{~g} / \mathrm{day}$ in males, $>5-25 \mathrm{~g} /$ day in females and $>10-50 \mathrm{~g} / \mathrm{d}$ in males, $>25 \mathrm{~g} /$ day in females and $>50 \mathrm{~g} /$ day in males), smoking status (non-smoker, ex-smoker, current smoker), BMI (continuous), physical activity (METs-h/week as continuous), prevalent hypertension, hypertriglyceridemia, hypercholesterolemia, diabetes, cancer, depression and family history of CVD (yes/no), education level (graduate, master, doctorate) and smoking-pack-years (continuous). Model 3 additionally adjusted for squared BMI, napping (yes/no), watching television (h/day), sitting time (h/week), snacking between meals (yes/no) and following special diets (yes/no)

Bold HR and 95\% CI reflects a significant result $(P<0.05)$

$H R$ hazard ratio, $C I$ confidence interval, $Q$ quintile, Ref. reference

${ }^{\mathrm{a}}$ Test for lineal trend calculated for the 5 quintiles

CVD risk was observed for each 5-unit increment of the PaleoDiet score (model 3).

We repeated Cox regression analysis after alternatively excluding one dietary component of the PaleoDiet score at a time (Table 4). No substantial changes were observed for these HRs compared to the HR calculated for the original PaleoDiet score except when the contribution of ultra-processed foods (as continuous variable per 5-unit increment), and when ultraprocessed foods, fruits and vegetables (comparison between extreme quintiles) were removed from the PaleoDiet score the inverse association between the PaleoDiet score and CVD was reduced, and the $P$ value was no longer statistically significant. Moreover, significant but weaker inverse associations were found for the alternative PaleoDiet scores without nuts or fish, and stronger association when cereal and grains did not negatively score (Table 4).

Subgroup analysis suggested no relevant differences according to levels of leisure-time physical activity, sex, or BMI ( $P$ for interaction $=0.730,0.616$ and 0.180 , respectively) (Supplemental Fig. 1).

Pearson correlation coefficients between the PaleoDiet score and MDS and MEDAS, were 0.32 and 0.48 , respectively. Figure 2 shows the standardized mean intake (servings/day) of the food groups used to create the PaleoDiet score in the nine categories of the joint combination of categories according to three groups of the PaleoDiet score and three groups of the MedDiet (MDS) score. The axis of the radar plot represents the mean consumption per day of each food group in units of SDs, being -0.8 the minimum value and +1 the maximum value. Figure $2 \mathrm{c}, \mathrm{f}$ and $\mathrm{i}$ show that among participants with the highest adherence to the PaleoDiet (Q5), all positive items associated with the Paleodiet, but also legumes and cereals and grains, increased with a higher adherence to the MedDiet. Moreover, Table 5 shows that the strongest inverse association with CVD risk was observed among participants with the highest quintile for both the PaleoDiet and the MedDiet scores $(P$ for trend $<0.001)$. When we applied the joint analysis of the PaleoDiet and the MEDAS scores, we also observed an inverse association with CVD risk among participants with the highest quintile for both scores compared to participants with the lowest adherence to both diets. (HR 0.51; 95\% CI $0.28,0.92 ; P$ for trend $=0.004)($ Supplemental Table 3$)$.

Lastly, results from sensitivity analyses did not substantially change except when we applied different total energy intake limits to exclude participants $(<\mathrm{P} 1$ and $>$ P99, total energy intake $<1073$ or $>3777 \mathrm{kcal}$ ) and when we excluded participants younger than 40 years (Supplemental Table 4). In these two cases, CVD risk was reduced in $65 \%$ and $43 \%$, respectively, in the fifth quintile as compared to the lowest quintile.

\section{Discussion}

This prospective Mediterranean cohort study, conducted among young adult participants, showed an inverse association between higher adherence to the PaleoDiet and CVD risk. Among participants with the highest PaleoDiet score (upper quintile), a 55\% relative CVD risk reduction was observed as compared to participants in the lowest 
Table 4 Hazard ratios and confidence intervals after alternate subtraction of each of its dietary components among participants of the SUN cohort

\begin{tabular}{|c|c|c|c|}
\hline & $\begin{array}{l}\mathrm{HR}^{\mathrm{a}}(95 \% \mathrm{CI}) \\
\text { per 5-unit increment }\end{array}$ & $\begin{array}{l}\mathrm{HR}^{\mathrm{b}}(95 \% \mathrm{CI}) \\
\text { Q5 vs. Q1 (Ref.) }\end{array}$ & $P$ for trend ${ }^{c}$ \\
\hline Overall & $0.81(0.68-0.96)^{*}$ & $0.45(0.27,0.76)^{* *}$ & 0.007 \\
\hline \multicolumn{4}{|c|}{ Excluding one item with a positive punctuation in the PaleoDiet score } \\
\hline PaleoDiet score without eggs & $0.81(0.68-0.97)^{*}$ & $0.41(0.24-0.70)^{* * *}$ & 0.008 \\
\hline PaleoDiet score without vegetables & $0.82(0.67-0.99) *$ & $0.62(0.36-1.09)$ & 0.080 \\
\hline PaleoDiet score without fruits & $0.83(0.69-1.00)^{*}$ & $0.67(0.39-1.15)$ & 0.104 \\
\hline PaleoDiet score without nuts & $0.79(0.66-0.96)^{*}$ & $0.55(0.31-0.96)^{*}$ & 0.032 \\
\hline PaleoDiet score without fish & $0.81(0.67-0.98)^{*}$ & $0.54(0.32-0.91)^{*}$ & 0.011 \\
\hline PaleoDiet score without unprocessed meats & $0.80(0.66-0.69) *$ & $0.47(0.28-0.79)^{* * *}$ & 0.009 \\
\hline \multicolumn{4}{|c|}{ Excluding one item with a negative punctuation in the PaleoDiet score } \\
\hline PaleoDiet score without cereals and grains & $0.76(0.63-0.91)^{* *}$ & $0.40(0.23-0.68)^{* *}$ & 0.001 \\
\hline PaleoDiet score without dairy products & $0.82(0.68-0.98)^{*}$ & $0.53(0.31-0.90)^{*}$ & 0.030 \\
\hline PaleoDiet score without legumes & $0.81(0.68-0.96)^{*}$ & $0.47(0.28-0.78)^{* *}$ & 0.011 \\
\hline PaleoDiet score without ultra-processed foods & $0.85(0.71-1.02)$ & $0.64(0.38-1.08)$ & 0.131 \\
\hline PaleoDiet score without culinary ingredients & $0.78(0.64-0.94) * *$ & $0.53(0.31-0.90) *$ & 0.010 \\
\hline
\end{tabular}

$H R$ hazard ratio, $C I$ confidence interval, $Q$ quintile, $R e f$. reference

$* P<0.05$

$* * P<0.01$

a Originally estimated logarithms of hazard ratios were multiplied by 50/55 and then exponentiated to correct for 50-point scale

${ }^{\mathrm{b}}$ Hazard ratio adjusted for age (10 groups) as the underlying variable of time, sex, year entering the cohort (1999-2001, 2002-2004, 2005-2007, 2008-2010, 2011-2014, 2015-2017), total energy intake (continuous), alcohol intake (teetotaler, $>0-5 \mathrm{~g} /$ day in females and $>0-10 \mathrm{~g} /$ day in males, $>5-25 \mathrm{~g} /$ day in females and $>10-50 \mathrm{~g} /$ day in males, $>25 \mathrm{~g} /$ day in females and $>50 \mathrm{~g} /$ day in males), smoking status (non-smoker, ex-smoker, current smoker), BMI (continuous), physical activity (METs-h/week as continuous), prevalent hypertension, hypertriglyceridemia, hypercholesterolemia, diabetes, cancer, depression and family history of CVD (yes/no), education level (graduate, master, doctorate) and smoking-pack-years (continuous), squared BMI, napping (yes/no), watching television (h/ day), sitting time (h/week), snacking between meals (yes/no) and following special diets (yes/no)

Bold HR and 95\% CI reflects a significant result $(P<0.05)$

${ }^{\mathrm{c}}$ Test for lineal trend calculated for the five quintiles quintile. This association was not significantly modified by sex, weight status or physical activity, and the results were robust in multiple sensitivity analyses aimed at controlling for residual confounding. A similar association was also observed when we alternatively excluded items one by one from the PaleoDiet score, although the consumption of fruits and vegetables, and the avoidance of ultra-processed foods may be key components of this diet. However, weaker inverse associations were also found when nuts or fish were excluded, suggesting the synergistic effect of all components within the PaleoDiet score. However, the prohibition of grains and cereals should be further explored since a stronger inverse association was found when this limitation was not part of the PaleoDiet score. Finally, in the joint analysis according to levels of adherence to PaleoDiet and MedDiet, the strongest inverse association with CVD was found among participants with the highest adherence to both dietary patterns.

Our findings are consistent with those published in a previous cohort study supporting the inverse association between the PaleoDiet and CVD death. In the REGARDS cohort study, with 21,423 participants and 863 CVD deaths, cardiovascular death risk was reduced by $22 \%$ in participants who most adhered to the PaleoDiet score (Q5) compared to the lowest quartile, although a borderline significance was observed [19]. In this study, only a stronger inverse association was found for the MedDiet compared to the PaleoDiet for total and specific causes of death. The Moli-sani cohort study, which used a similar PaleoDiet definition as Whalen and cols. [37], found a significant association between the PaleoDiet and total death but no significant association with cardiovascular mortality [20]. In that study, no modification effect of the PaleoDiet was observed in a stratified analysis by level of adherence to the MedDiet [20]. In another study, a score comprising dietary habits and other lifestyle behaviors that could be concordant with a PaleoDiet lifestyle such as limited alcohol consumption, not smoking, high levels of physical activity, and low levels of sedentary behavior showed an inverse relationship with all CVD mortality [38]. 

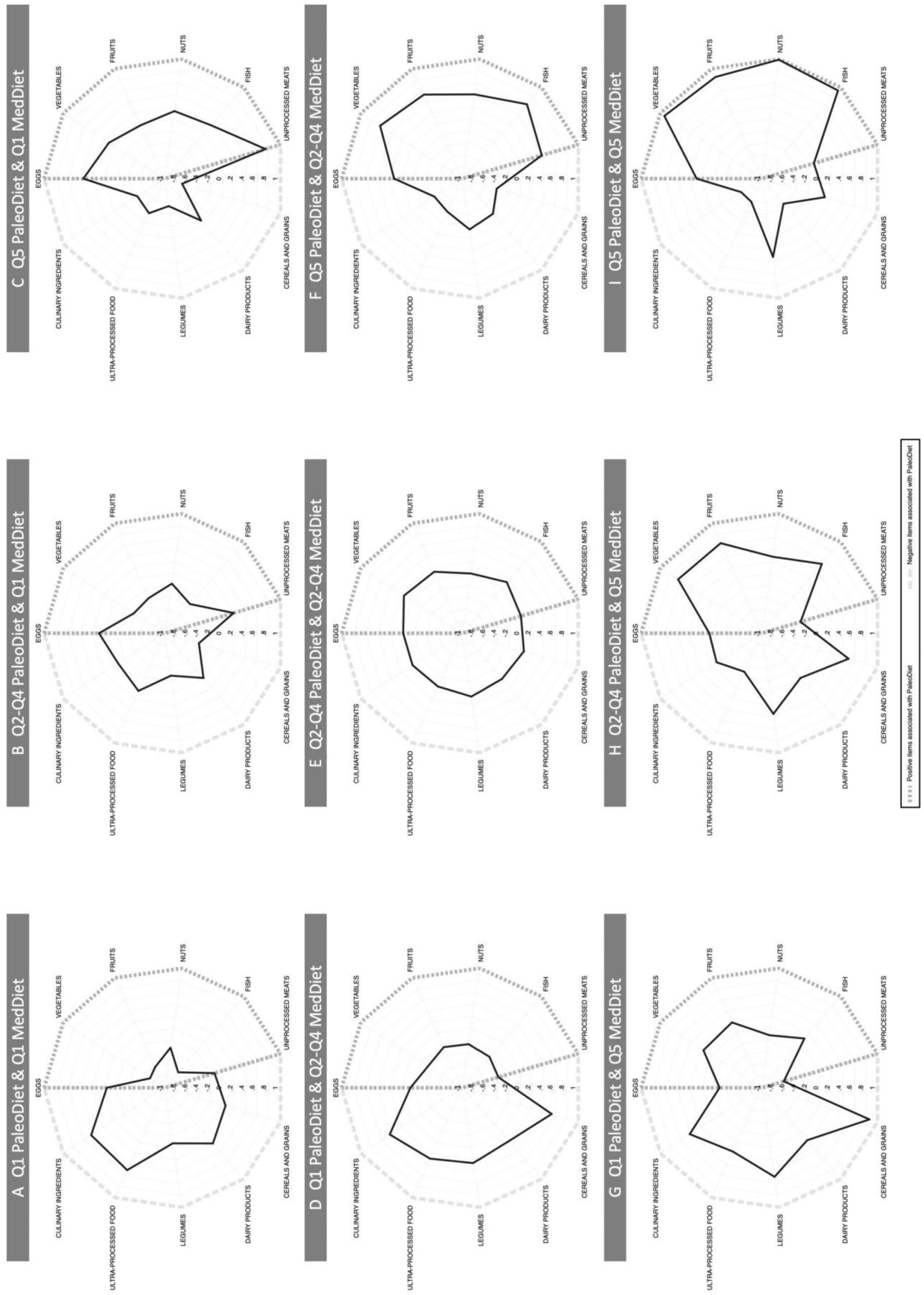
4 Fig. 2 Mean intake serv/day (per SDs) of common group foods in both patterns according to the categories of joint analyses of the recategorized PaleoDiet and MedDiet scores (MDS, (28)). C Category. The radar plot axis is expressed in standard deviations (SD). $1-\mathrm{SD}$ of eggs is equivalent to 1.5 serv/day; 1 -SD of vegetables is equivalent to 1.6 serv/day; $1-\mathrm{SD}$ of fruits is equivalent to $1.2 \mathrm{serv} /$ day; 1 -SD of nuts is equivalent to 0.6 serv/day; 1 -SD of fish is equivalent to $1.7 \mathrm{serv} /$ day; 1 -SD of unprocessed meats is equivalent to 2.1 serv/day; $1-\mathrm{SD}$ of cereals and grains is equivalent to $1.4 \mathrm{serv} /$ day; $1-\mathrm{SD}$ of dairy products is equivalent to $1.7 \mathrm{serv} /$ day; $1-\mathrm{SD}$ of legumes is equivalent to $1.3 \mathrm{serv} /$ day; 1 -SD of ultra-processed foods is equivalent to 1.9 serv/day; $1-\mathrm{SD}$ of culinary ingredients is equivalent to 2.1 serv/day

The inverse association between the PaleoDiet and CVD risk is supported by the previously reported effect of the PaleoDiet on different markers of CVD risk [18]. The PaleoDiet has shown a significant reduction in the risk of anthropometric markers such as body weight [12, 39-43], waist circumference [12, 39-42], BMI [12, 40, 41], and the percentage of fat mass [39, 42, 44]. Additionally, those who followed a PaleoDiet-style lowered their systolic and diastolic blood pressure, total blood cholesterol, triglycerides, LDL-cholesterol and had increased levels of HDL-cholesterol [12, 39-43]. However, most of these studies had a small sample size and short follow-up [17].

Table 5 Hazard ratios and confidence intervals according to joint classification by combined exposures to the PaleoDiet and the MedDiet score (MDS)

\begin{tabular}{cccc}
\hline \multicolumn{4}{c}{ Paleolithic diet score (min-max) } \\
\cline { 2 - 4 } & Q1 (16-28) & Q2-Q4 (29-36) & Q5 (37-50) \\
\hline \multicolumn{4}{c}{ Mediterranean diet score (min-max) } \\
Q1 (0-3) & 1 (Ref.) & $0.64(0.34-$ & $0.52(0.17-1.53)$ \\
& & $1.20)$ & \\
Q2-Q4 & $0.73(0.38-$ & $\mathbf{0 . 4 8}(\mathbf{0 . 2 6}-$ & $\mathbf{0 . 3 6}(\mathbf{0 . 1 8}-\mathbf{0 . 7 4})$ \\
(4-6) & $1.42)$ & $\mathbf{0 . 8 6})$ & \\
Q5 (7-9) & $0.21(0.03-$ & $\mathbf{0 . 2 8}(\mathbf{0 . 1 2}-$ & $\mathbf{0 . 2 2}(\mathbf{0 . 0 8}-\mathbf{0 . 6 4})$ \\
& $1.61)$ & $\mathbf{0 . 6 2})$ & \\
\hline
\end{tabular}

MedDiet score (MDS) was assessed using the Trichopoulou's score [31]

Hazard ratio adjusted for age (10 groups) as the underlying variable of time, sex, year entering the cohort (1999-2001, 2002-2004, 2005-2007, 2008-2010, 2011-2014, 2015-2017), total energy intake (continuous), alcohol intake (teetotaler, $>0-5 \mathrm{~g} / \mathrm{d}$ in females and $>0-10 \mathrm{~g} /$ day in males, $>5-25 \mathrm{~g} /$ day in females and $>10-50 \mathrm{~g} /$ day in males, $>25 \mathrm{~g} /$ day in females and $>50 \mathrm{~g} /$ day in males), smoking status (non-smoker, ex-smoker, current smoker), BMI (continuous), physical activity (METs-h/week as continuous), prevalent hypertension, hypertriglyceridemia, hypercholesterolemia, diabetes, cancer, depression and family history of CVD (yes/no), education level (graduate, master, doctorate) and smoking-pack-years (continuous), squared BMI, napping (yes/no), watching television (h/day), sitting time (h/week), snacking between meals (yes/no) and following special diets (yes/no)

Bold HR and 95\% CI reflects a significant result $(P<0.05)$

$Q$ quintile, Ref. reference
The potential beneficial effects of the PaleoDiet for cardiovascular risk could be attributed to a high consumption of fruits, vegetables (important sources of fiber), fish and nuts, MUFAs and PUFAs, as well as to a limited consumption of ultra-processed foods, added sugar, salt and refined oils intake. Similar to traditional and ancient diets, the PaleoDiet promotes higher nutrient density [45] and higher fiber intake [46] compared to current Western diets [47]. In comparison with Western diets, in a previous study it was observed that PaleoDiet could contain 200-300\% more fiber, 150-200\% more PUFAs and MUFAs, $400 \%$ more omega-3 fatty acids, but $60-70 \%$ less SFAs [15].

Eaton and cols. estimated a high consumption of vegetable foods in Paleolithic diets and, according to this, the preagricultural fiber intake exceeded 100 g/day [46, 48]. Fiber consumed by humans during the Paleolithic area came primarily from fruits, legumes, nuts, and other noncereal vegetable sources, and its content of phytic acid would had been less than that of the fiber consumed now in industrialized nations, which comes largely from grains [48]. According to Eaton and Konner, the proportion of soluble, fermentable fiber relative to insoluble, non-fermentable fiber was likely higher in meals consumed by preagricultural humans [48]. In the current study, we only found 50\% more fiber in the lowest vs. highest PaleoDiet quintiles and there was only a small difference in SFA intake. The low mean SFAs intake found in our study is explained because the PaleoDiet score quintiles depend on the distribution of SFAs in our Mediterranean population which is relatively low [17, 49]. In addition, restriction of ultra-processed foods is associated with lower $\mathrm{Na}$ intake (400-500\% lower) and higher K intake (300-400\% higher) in comparison with the typical industrialized and Western diets [50, 51].

We observed a moderate correlation between the PaleoDiet score and two MedDiet indices. Moreover, a stronger inverse association of the PaleoDiet with CVD was observed when adherence to the MedDiet was highest. This finding means that although the PaleoDiet is proxy of a healthy eating model, the MedDiet may exert even higher cardiovascular benefits. Contrary to the MedDiet, the PaleoDiet recommends a low consumption of legumes and whole cereals and grains, and a high consumption of lean meat (white or red meat). Several studies have suggested that legume consumption improves multiple cardiovascular risk factors (blood pressure, LDL concentrations in blood, body weight) and protects against type 2 diabetes, reducing glycated hemoglobin levels in diabetic patients and improving insulin sensitivity $[52,53]$. In addition, the limitation of whole grains consumption in the PaleoDiet may hamper that key nutrients needs such as fiber, vitamins, minerals, lignans, and phytochemicals (phenolic acids, polyphenols, and phytosterol compounds) are met $[54,55]$. These nutrients 
have been positively associated with longevity, and lower risk of obesity, type 2 diabetes, heart disease and colon cancer [54-57]. Finally, there is still some controversy about the cardiovascular effect of unprocessed red meat $[58,59]$, but several recent analyses [60-62] suggested a positive association between the consumption of unprocessed red meats and cardiovascular risk. Moreover, no evidence from these studies suggests any cardiometabolic benefits of unprocessed red meat consumption [59].

This study has some limitations. First, the SUN cohort participants did not actively choose a lifestyle according to a PaleoDiet, since we applied an a posteriori classification criterion using a self-reported semi-quantitative FFQ. A randomized intervention study with long-term follow-up will be needed to assess the effect of an increased adherence to the PaleoDiet, and also to other lifestyle factors associated with this diet, on cardiovascular risk. Second, we used a self-reported semi-quantitative FFQ, which is susceptible to non-differential measurement error, which would more likely underestimate the true association. However, the FFQ is considered as the most appropriate and practical approach to assess usual food consumption in large cohorts [22]. Third, our participants were young adults with low prevalence of cardiovascular risk factors and this explains the small number of observed cardiovascular events, especially among women. Further analyses in larger studies are needed to confirm our results and to explore potential interactions with variables such age, sex or other lifestyles such as physical activity. Fourth, the SUN cohort did not collect biomarkers of cardiovascular risk factors and therefore we were not able to identify potential mechanisms that could explain the inverse association between the PaleoDiet and the risk of CVD. Five, the FFQ was not specifically designed to collect data about the new NOVA classification of ultra-processed foods consumption. We could not include some items (cereal and energy bars, energy drinks, health and slimming products, and meat or vegetable nuggets) because the FFQ of the SUN project did not include these items. Therefore, there is the potential for some degree of misclassification of ultraprocessed food consumption inherent in our methodology. However, our FFQ was previously validated and represents the main foods ingested by the studied population [23], including ultra-processed foods and this potential misclassification would be non-differential according to the status of participants at the end of the study. Six, we cannot rule out the existence of residual confounding, although we adjusted for the well-known risk factors of CVD in different multivariable models and the results were very similar regardless of the variables used for adjustment. Seven, another limitation is the difficulty of measuring the PaleoDiet, although we used a new index based on our previous comprehensive review [17]. Current adaptations of the PaleoDiet may vary from some archeological records [63] and, for example, although potato intake was not included in our score, some studies suggest that root vegetables with high starch content may be a component of Paleolithic diets [64]. Finally, the external validity of our results is limited since participants in our cohort were relatively young with high educational level and with low prevalence of cardiovascular risk factors, which limits the generalization of the results to the general population. However, lack of representativeness does not necessarily imply lack of validity, and the inclusion of highly educated participants in our cohort improves the quality of self-reported information and reduces the possible confounding by educational level and other socio-economic factors [22]. In addition, the characteristics of our cohort do not prevent from establishing associations that can be generalized to other groups, as long as similar biological mechanisms are plausible in these populations.

Strengths of our study are the high retention rate $(91 \%)$, the blind confirmation of CVD events by a cardiologist, which minimizes the potential for misclassification, the prospective design, which limits the possibility of reverse causality, the ability to control for a large number of potential confounders and the long-term follow-up. Lastly, we conducted a wide array of sensitivity analyses to test the robustness of our results.

In conclusion, our results suggest that the PaleoDiet may decrease CVD risk in young adult participants from a Mediterranean country. This association could be explained by the synergistic effect of all the items of the PaleoDiet score although low consumption of ultraprocessed foods, and high consumption of fruits and vegetables seem to be key components to reduce CVD risk. A slightly higher inverse association was also found when cereals and grains were not negatively scored in the PaleoDiet. Moreover, a stronger inverse association with the PaleoDiet was found as the level of adherence to the MedDiet increased. Further research with different populations and longer follow-up is needed to replicate these findings and to better clarify the health impact that restriction of typical Mediterranean foods, such as legumes and whole grains, may have on the prevention of CVD for the general population.

Supplementary Information The online version contains supplementary material available at https://doi.org/10.1007/s00394-021-02696-9.

Acknowledgements We thank very specially all participants in the SUN cohort for their long-standing and enthusiastic collaboration and our advisors from Harvard TH Chan School of Public Health Walter Willett, Alberto Ascherio, Frank B. Hu and Meir J. Stampfer who helped us to design the SUN Project, the PREDIMED study and the PREDIMED-PLUS on-going trial. We thank other members of the SUN Group: Alonso A, Álvarez-Álvarez I, Balaguer A, Barbagallo M, Barrientos I, Barrio-López MT, Basterra-Gortari FJ, Battezzati A, Bazal P, Benito S, Bertoli S, Beulen Y, Beunza JJ, Buil-Cosiales P, Canales M, Carlos S, Carmona L, Cervantes S, Cristobo C, de Irala J, 
de la Fuente-Arrillaga C, de la Rosa PA, Delgado-Rodríguez M, DíazGutiérrez J, Díez Espino J, Domínguez L, Donat-Vargas C, Donazar M, Eguaras S, Fernández-Montero A, Fresán U, Galbete C, GarcíaArellano A, García López M, Gardeazábal I, Gutiérrez-Bedmar M, Goméz-Domingos AL, Gómez-Donoso C, Gómez-Gracia E, Goñi E, Guillén F, Henríquez P, Hernández A, Hershey MS, Hidalgo-Santamaría M, Hu E, Lahortiga F, Leone A, Llorca J, López del Burgo C, Marí A, Marques I, Martí A, Martín-Moreno JM, Martínez-Lapiscina EH, Mendonça R, Menéndez C, Molendijk M, Molero P, Murphy K, Muñoz M, Núñez-Córdoba JM, Pajares R, Papadaki A, Parletta N, Pérez de Ciriza P, Pérez Cornago A, Pérez de Rojas J, Pimenta AM, Pons J, Ramallal R, Razquin C, Rico A, Romanos A, Ruano C, Ruiz L, Ruiz Zambrana A, Salgado E, San Julián B, Sánchez D, Sánchez-Bayona R, Sánchez-Tainta A, Sánchez-Villegas A, Sayón-Orea C, Schlatter J, Serrano-Martinez M, Toledo E, Toledo J, Tortosa A, Valencia F, Vázquez Z, Zarnowiecki D.

Author contributions VO, IZ and MR-C designed research; VO, MR-C, IZ and MAM-G conducted research; VO, MR-C and MAM-G analyzed data and performed statistical analysis; VO wrote paper; MR-C and IZ had primary responsibility for final content; MR-C, IZ, JAM, NM-C, LG, SS and MB-R helped with the methodology. All authors reviewed and approved the manuscript.

Funding Open Access funding provided thanks to the CRUE-CSIC agreement with Springer Nature. The SUN Project has received funding from the Instituto de Salud Carlos III and European Regional Development Fund (FEDER) (RD 06/0045, CIBER-OBN, Grants PI10/02658, PI10/02293, PI13/00615, PI14/01668, PI14/01798, PI14/01764, PI17/01795, PI20/00564 and G03/140), the Navarra Regional Government (45/2011, 122/2014, 41/2016), and the University of Navarra.

Availability of data and material This study uses data from the Seguimiento Universidad de Navarra (SUN) cohort. All data and materials as well as software application or custom code used during the current study shall be made available from the corresponding author on reasonable request.

Code availability Not applicable.

\section{Declarations}

Conflict of interest All authors declared no conflicts of interest to disclose.

Ethics approval Participants received written information about the specific data required in the questionnaires, the protection to safeguard their privacy, and the future feedback from the research team. We also informed the potential candidates of their right to refuse to participate in the SUN study or to withdraw their consent to participate at any time without reprisal, according to the principles of the Declaration of Helsinki. The Research Ethics Committee of the University of Navarra approved this method to request the informed consent of participants. This cohort is registered at clinicaltrials.gov as NCT02669602.

Consent to participate All participants gave written, informed consent at enrollment.

Consent for publication Not applicable.

Open Access This article is licensed under a Creative Commons Attribution 4.0 International License, which permits use, sharing, adaptation, distribution and reproduction in any medium or format, as long as you give appropriate credit to the original author(s) and the source, provide a link to the Creative Commons licence, and indicate if changes were made. The images or other third party material in this article are included in the article's Creative Commons licence, unless indicated otherwise in a credit line to the material. If material is not included in the article's Creative Commons licence and your intended use is not permitted by statutory regulation or exceeds the permitted use, you will need to obtain permission directly from the copyright holder. To view a copy of this licence, visit http://creativecommons.org/licenses/by/4.0/.

\section{References}

1. Timmis A, Townsend N, Gale CP et al (2020) European society of cardiology: cardiovascular disease statistics 2019. Eur Heart J 41:12-85. https://doi.org/10.1093/eurheartj/ehz859

2. WHO (World Health Organ) (2017) Cardiovascular diseases (CVDs). https://www.who.int/news-room/fact-sheets/detail/cardi ovascular-diseases-(cvds). Accessed 2 Sept 2021

3. Myers L, Mendis S (2014) Cardiovascular disease research output in WHO priority areas between 2002 and 2011. J Epidemiol Glob Health 4(1):23-28. https://doi.org/10.1016/j.jegh.2013.09.007

4. Ko H, Lee J, Shin J, Jo E (2015) Health-related quality of life and cardiovascular disease risk in Korean adults. Korean J Fam Med 36:349-356. https://doi.org/10.4082/kjfm.2015.36.6.349

5. Gheorghe A, Griffiths U, Murphy A et al (2018) The economic burden of cardiovascular disease and hypertension in low- and middle-income countries: a systematic review. BMC Public Health 18:975. https://doi.org/10.1186/s12889-018-5806-x

6. Turco JV, Inal-Veith A, Fuster V (2018) Cardiovascular health promotion: an issue that can no longer wait. J Am Coll Cardiol 72:908-913. https://doi.org/10.1016/j.jacc.2018.07.007

7. Mozaffarian D, Appel LJ, van Horn L (2011) Components of a cardioprotective diet. Circulation 123:2870-2891. https://doi.org/ 10.1161/CIRCULATIONAHA.110.968735

8. Martínez-González MA, Gea A, Ruiz-Canela M (2019) The Mediterranean diet and cardiovascular health. Circ Res 124:779-798. https://doi.org/10.1161/CIRCRESAHA.118.313348

9. Yu E, Malik VS, Hu FB (2018) Cardiovascular disease prevention by diet modification: JACC health promotion series. J Am Coll Cardiol 72:914-926. https://doi.org/10.1016/j.jacc.2018. 02.085

10. Bocherens H (2009) Neanderthal dietary habits: review of the isotopic evidence. Evol Hominin Diets. https://doi.org/10.1007/ 978-1-4020-9699-0_19

11. Andrikopoulos S (2016) The paleo diet and diabetes: studies are inconclusive about the benefits of the paleo diet in patients with type 2 diabetes. Med J Aust 205:151-152. https://doi.org/10.5694/ mja16.00347

12. Jönsson T, Granfeldt Y, Ahrén B et al (2009) Beneficial effects of a Paleolithic diet on cardiovascular risk factors in type 2 diabetes: a randomized cross-over pilot study. Cardiovasc Diabetol 8:35. https://doi.org/10.1186/1475-2840-8-35

13. Limdi JK (2018) Dietary practices and inflammatory bowel disease. Indian J Gastroenterol 37:284-292. https://doi.org/10.1007/ s12664-018-0890-5

14. Manheimer EW, van Zuuren EJ, Fedorowicz Z, Pijl H (2015) Paleolithic nutrition for metabolic syndrome: systematic review and meta-analysis. Am J Clin Nutr 102:922-932. https://doi.org/ 10.3945/ajen.115.113613

15. O'Keefe JH, Cordain L (2004) Cardiovascular disease resulting from a diet and lifestyle at odds with our Paleolithic genome: how to become a 21st-century Hunter-Gatherer. Mayo Clin Proc 79:101-108. https://doi.org/10.4065/79.1.101 
16. Eaton SB, Konner M, Shostak M (1988) Stone agers in the fast lane: chronic degenerative diseases in evolutionary perspective. Am J Med 84:739-749. https://doi.org/10.1016/0002-9343(88) 90113-1

17. de la O V, Zazpe I, Martínez JA et al (2021) Scoping review of Paleolithic dietary patterns: a definition proposal. Nutr Res Rev 34:78-106. https://doi.org/10.1017/S0954422420000153

18. Ghaedi E, Mohammadi M, Mohammadi $\mathrm{H}$ et al (2019) Effects of a Paleolithic diet on cardiovascular disease risk factors: a systematic review and meta-analysis of randomized controlled trials. Adv Nutr (Bethesda, Md) 10:634-646. https://doi.org/10.1093/advan ces/nmz007

19. Whalen KA, Judd S, McCullough ML et al (2017) Paleolithic and Mediterranean diet pattern scores are inversely associated with all-cause and cause-specific mortality in adults. J Nutr 147:612620. https://doi.org/10.3945/jn.116.241919

20. Bonaccio M, di Castelnuovo A, Costanzo S et al (2021) Association of a traditional Mediterranean diet and non-Mediterranean dietary scores with all-cause and cause-specific mortality: prospective findings from the Moli-sani Study. Eur J Nutr 60:729746. https://doi.org/10.1007/s00394-020-02272-7

21. Carlos S, de La Fuente-Arrillaga C, Bes-Rastrollo M et al (2018) Mediterranean diet and health outcomes in the SUN cohort. Nutrients. https://doi.org/10.3390/nu10040439

22. Willett WC (2013) Nutritional epidemiology, 3rd edn. Oxford University Press, New York

23. Martin-Moreno JM, Boyle P, Gorgojo L et al (1993) Development and validation of a food frequency questionnaire in Spain. Int $\mathrm{J}$ Epidemiol 22:512-519

24. Fernández-Ballart JD, Piñol JL, Zazpe I et al (2010) Relative validity of a semi-quantitative food-frequency questionnaire in an elderly Mediterranean population of Spain. Br J Nutr 103:18081816. https://doi.org/10.1017/S0007114509993837

25. Moreiras O, Carbajal A, Cabrera L (2005) Tablas de composición de alimentos, Pirámide. Madrid. https://www.sennutricion.org/es/ 2013/05/14/tablas-de-composicin-de-alimentos-moreiras-et-al. Accessed 2 Sept 2021

26. Mataix Verdú J (2003) Tabla de composición de alimentos españoles (Spanish Food Composition Table), 4th edn. Universidad de Granada, Granada

27. Monteiro CA, Cannon G, Lawrence M et al (2019) Ultra-processed foods, diet quality, and health using the NOVA classification system. FAO, Rome. http://www.fao.org/3/ca5644en/ca564 4en.pdf. Accessed 2 Sept 2021

28. Rico-Campà A, Martínez-González MA, Alvarez-Alvarez I et al (2019) Association between consumption of ultra-processed foods and all cause mortality: SUN prospective cohort study. BMJ 365:11949. https://doi.org/10.1136/bmj.11949

29. de Mendonça RD, Lopes ACS, Pimenta AM et al (2017) Ultraprocessed food consumption and the incidence of hypertension in a Mediterranean cohort: the Seguimiento Universidad de Navarra Project. Am J Hypertens 30:358-366. https://doi.org/10.1093/ajh/ hpw137

30. Gómez-Donoso C, Sánchez-Villegas A, Martínez-González MA et al (2020) Ultra-processed food consumption and the incidence of depression in a Mediterranean cohort: the SUN Project. Eur J Nutr. https://doi.org/10.1007/s00394-019-01970-1

31. Trichopoulou A, Costacou T, Bamia C, Trichopoulos D (2003) Adherence to a Mediterranean diet and survival in a Greek population. N Engl J Med 348:2599-2608. https://doi.org/10.1056/ NEJMoa025039

32. Schröder H, Fitó M, Estruch R et al (2011) A short screener is valid for assessing Mediterranean diet adherence among older Spanish men and women. J Nutr 141:1140-1145. https://doi.org/ 10.3945/jn. 110.135566
33. Li J, Guasch-Ferré M, Chung W et al (2020) The Mediterranean diet, plasma metabolome, and cardiovascular disease risk. Eur Heart J 41:2645-2656. https://doi.org/10.1093/eurheartj/ehaa209

34. Hernán MA, Robins JM (2020) Causal inference. https://www. cdn1.sph.harvard.edu/wp-content/uploads/sites/1268/2021/03/ ciwhatif_hernanrobins_30mar21.pdf. Accessed 2 Sept 2021

35. Trichopoulou A, Bamia C, Trichopoulos D (2009) Anatomy of health effects of Mediterranean diet: Greek EPIC prospective cohort study. BMJ (Clin Res Ed) 338:b2337. https://doi.org/10. 1136/bmj.b2337

36. Saary MJ (2008) Radar plots: a useful way for presenting multivariate health care data. J Clin Epidemiol 61:311-317

37. Whalen KA, McCullough M, Flanders WD et al (2014) Paleolithic and Mediterranean diet pattern scores and risk of incident, sporadic colorectal adenomas. Am J Epidemiol 180:1088-1097. https://doi.org/10.1093/aje/kwu235

38. Troeschel AN, Hartman TJ, Flanders WD et al (2021) A novel evolutionary-concordance lifestyle score is inversely associated with all-cause, all-cancer, and all-cardiovascular disease mortality risk. Eur J Nutr 60:3485-3497. https://doi.org/10.1007/ s00394-021-02529-9

39. Lindeberg S, Jönsson T, Granfeldt Y et al (2007) A Palaeolithic diet improves glucose tolerance more than a Mediterranean-like diet in individuals with ischaemic heart disease. Diabetologia 50:1795-1807. https://doi.org/10.1007/s00125-007-0716-y

40. Mellberg C, Sandberg S, Ryberg M et al (2014) Long-term effects of a Palaeolithic-type diet in obese postmenopausal women: a 2-year randomized trial. Eur J Clin Nutr 68:350-357. https://doi. org/10.1038/ejcn.2013.290

41. Boers I, Muskiet FA, Berkelaar E et al (2014) Favourable effects of consuming a Palaeolithic-type diet on characteristics of the metabolic syndrome: a randomized controlled pilot-study. Lipids Health Dis 13:160. https://doi.org/10.1186/1476-511X-13-160

42. Genoni A, Lyons-Wall P, Lo J, Devine A (2016) Cardiovascular, metabolic effects and dietary composition of ad-libitum Paleolithic vs. Australian guide to healthy eating diets: a 4-week randomised trial. Nutrients. https://doi.org/10.3390/nu8050314

43. Masharani U, Sherchan P, Schloetter M et al (2015) Metabolic and physiologic effects from consuming a hunter-gatherer (Paleolithic)-type diet in type 2 diabetes. Eur J Clin Nutr 69:944948. https://doi.org/10.1038/ejcn.2015.39

44. Stomby A, Simonyte K, Mellberg C et al (2005) (2015) Dietinduced weight loss has chronic tissue-specific effects on glucocorticoid metabolism in overweight postmenopausal women. Int J Obes 39:814-819. https://doi.org/10.1038/ijo.2014.188

45. Chenard CA, Rubenstein LM, Snetselaar LG, Wahls TL (2019) Nutrient composition comparison between a modified Paleolithic diet for multiple sclerosis and the recommended healthy US-style eating pattern. Nutrients. https://doi.org/10.3390/ nu11030537

46. Eaton SB, Eaton SB, Konner MJ (1997) Paleolithic nutrition revisited: a twelve-year retrospective on its nature and implications. Eur J Clin Nutr 51:207-216. https://doi.org/10.1038/sj.ejcn.16003 89

47. Cordain L, Eaton SB, Sebastian A et al (2005) Origins and evolution of the Western diet: health implications for the 21 st century. Am J Clin Nutr 81:341-354. https://doi.org/10.1093/ajcn.81.2.341

48. Eaton S, Konner M (1997) Review Paleolithic nutrition revisited: a twelve-year retrospective on its nature and implications. Eur J Clin Nutr. https://doi.org/10.1038/sj.ejcn.1600389

49. Cordain L, Miller JB, Eaton SB et al (2000) Plant-animal subsistence ratios and macronutrient energy estimations in worldwide hunter-gatherer diets. Am J Clin Nutr 71:682-692. https://doi.org/ 10.1093/ajcn/71.3.682 
50. Eaton SB, Eaton SB (2000) Paleolithic vs. modern diets-selected pathophysiological implications. Eur J Nutr 39:67-70. https://doi. org/10.1007/s003940070032

51. Eaton SB, Nelson DA (1991) Calcium in evolutionary perspective. Am J Clin Nutr 54:281S-287S. https://doi.org/10.1093/ajcn/54.1. $281 \mathrm{~S}$

52. Anand SS, Hawkes C, de Souza RJ et al (2015) Food consumption and its impact on cardiovascular disease: importance of solutions focused on the globalized food system. J Am Coll Cardiol 66:1590-1614. https://doi.org/10.1016/j.jacc.2015.07.050

53. Willett W, Rockström J, Loken B et al (2019) Food in the Anthropocene: the EAT-Lancet Commission on healthy diets from sustainable food systems. Lancet 393:447-492. https://doi.org/10. 1016/S0140-6736(18)31788-4

54. McRae MP (2017) Health benefits of dietary whole grains: an umbrella review of meta-analyses. J Chiropr Med 16:10-18. https://doi.org/10.1016/j.jcm.2016.08.008

55. Wu H, Flint AJ, Qi Q et al (2015) Association between dietary whole grain intake and risk of mortality. JAMA Intern Med 175:373-384. https://doi.org/10.1001/jamainternmed.2014.6283

56. Jawhara M, Sørensen SB, Heitmann BL, Andersen V (2019) Biomarkers of whole-grain and cereal-fiber intake in human studies: a systematic review of the available evidence and perspectives. Nutrients 11:2994. https://doi.org/10.3390/nu11122994

57. Cho SS, Qi L, Fahey GC, Klurfeld DM (2013) Consumption of cereal fiber, mixtures of whole grains and bran, and whole grains and risk reduction in type 2 diabetes, obesity, and cardiovascular disease. Am J Clin Nutr 98:594-619. https://doi.org/10.3945/ajcn. 113.067629

58. Qian F, Riddle MC, Wylie-Rosett J, Hu FB (2020) Red and processed meats and health risks: how strong is the evidence? Diabetes Care. https://doi.org/10.2337/dci19-0063

59. Johnston BC, Zeraatkar D, Han MA et al (2019) Unprocessed red meat and processed meat consumption: dietary guideline recommendations from the Nutritional Recommendations (NutriRECS) Consortium. Ann Intern Med. https://doi.org/10.7326/M19-1621

60. Wang X, Lin X, Ouyang YY et al (2016) Red and processed meat consumption and mortality: dose-response meta-analysis of prospective cohort studies. Public Health Nutr 19:893-905. https:// doi.org/10.1017/S1368980015002062

61. Clark MA, Springmann M, Hill J, Tilman D (2019) Multiple health and environmental impacts of foods. Proc Natl Acad Sci. https://doi.org/10.1073/pnas.1906908116

62. Guasch-Ferré M, Satija A, Blondin SA et al (2019) Meta-analysis of randomized controlled trials of red meat consumption in comparison with various comparison diets on cardiovascular risk factors. Circulation. https://doi.org/10.1161/CIRCULATIONAHA. 118.035225

63. Copeland L, Hardy K (2018) Archaeological starch. Agronomy 8:4. https://doi.org/10.3390/agronomy8010004

64. Hardy K, Brand-Miller J, Brown KD et al (2015) The Importance of dietary carbohydrate in human evolution. Q Rev Biol. https:// doi.org/10.1086/682587 